\title{
The Effect of Bobath Concept and Conventional Approach on the, Functional Outcome in the Post Stroke Hemiplegic Individuals
}

\author{
Dr G Varadharajulu ${ }^{1}$, Dr. Lathika Shetty ${ }^{2}$, Dr. Kulamani Sahoo ${ }^{3}$ \\ (Dean, Faculty of physiotherapy, KIMSDU, Karad) \\ (Professor in Radiology, K S Hegade Hospital, NITTE university, Manglore) \\ (Professor, Head, Department of radiology, Krishna hospital, KIMSDU, Karad.)
}

\begin{abstract}
:
Introduction: Stroke caused by cerebro vascular accident producing the symptom of hemiplegia has been therapeutically managed by many approaches resulting to satisfactory functional outcome without knowing the significant of each approach being used. So in this study reasearcher tried to find the significant effects of two common approaches which are in practiced at present world wide.These approaches are bobath approach of stroke rehabilitation and conventional approaches.

Objective: Tried to find significant difference in the outcome in both the approaches beore and after the treatment.

Method: pre post experimental design of study included total 60 patients who fulfills the inclusion criteria and treated with above mentioned approaches the functional outcomes were assessed by using modified barthel index which has got highest value of inter and intra rater reliability. Result: there was no statistically significant difference between group $A$ and group $B(p=0.0002)$. With in group pre post analysis were highy significant $(p<0.0001)$.

Conclusion: Based on the statistical analysis and interpretations the study accepts the null hypothesis but in the group B (experimental group) the functional recovery attained by the clients were quiet earlier than Group A (control group)
\end{abstract}

Keyword: Bobath, Modified Barthel Index

\section{Introduction}

Stroke is a focal neurological deficit, secondary to cerebro vascular accident causing transient or permanent damage to the affected part of brain, as clinical manifestations there is a paralysis of one half of the body always contra lateral to site of lesion.There are numerous approaches are being in practice of physiotherapy for the rehabilitation of stroke patients but very few are proven better in functional outcome. To evidence this as such there is no studies on any approach which has got significant effect of structural changes along with functional outcome in this population.

So in this study the researchers, tried to find out initially the functional outcome by using the commonly used therapeutic approaches such as Bobath concept of stroke rehabilitation and conventional approach of stroke rehabilitation to find out functional out come followed by co- relation with morphological changes at the site of lesion. Both the approaches contradictory to each other in following two ways

1. Principles of application

2. The time of intervention.

Bobath concept:

The Bobath concept of stroke rehabilitation is a very much ancient technique implemented for the early recovery of stroke patients predominantly in Western countries(UK, US and Canada). In recent decades this approach is widely being accepted and considered as an important approach for stroke rehabilitation in India also. The principles of Bobath concept were framed on the basis of predicted clinical manifestations of stroke patients from the day one of the incidence of stroke. On the basis of this the following principles are framed.

1. Normal postural alignment

2. Inhibition versus facilitation

3. Assessment versus treatment

So all this princilples are being used from the very early phase of incidence of stroke to final stage of recovery( Brunstrom stage 6)

Conventional approach: 
The conventional approach of stroke rehabilitation mainly given as a symptomatic management of stroke survivors. It can be exiquted at any stage of recovery for any kind of clinical presentation.

In Indian scenario all the patients who have undergone treatment with above mentioned approaches were found highly benefited (based on the literature review) because in India stroke patients are generally referred to physiotherapist once the signs and symptoms of stroke were established. Those patients who are referred early were being treated using Bobath concept of stroke rehabilitation and those who are late there is no other way except going for conventional approach. But the patients are significantly improving on the functional outcome. But the functional recovery found at the affected limb are throwing light upon the young researchers in the stroke rehabilitation that the significant functional outcome after any therapeutic approach will not only effective at the body but also it will have significant effect at site of lesion (based on evidence of neuronal plasticity). This input has given idea to the researcher of this study to find the morphological changes at the site of lesion by using both the above approaches initially assessing the functional outcome using MBI subsequently finding the site of changes at the site of lesion using functional MRI.

\section{Literature survey}

Amir H Bakhtiary and Elham Fatemy ${ }^{3}$ in there randomized controlled study-Does electrical stimulation reduces spasticity after stroke? To investigate the therapeutic effect of electrical stimulation on plantar flexor spasticity in stroke patients studied forty stroke patients (aged from 42 to 65 years) with ankle plantar flexor spasticity.Fifteen minutes of inhibitory Bobath techniques were applied to one experimental group and a combination of 9 minutes of electrical stimulation on the dorsiflexor muscles and inhibitory Bobath techniques was applied to another group for 20 sessions daily. Outcome measure were passive ankle joint dorsiflexion range of motion, dorsiflexion strength test, plantar flexor muscle tone by Modified Ashworth Scale and soleus muscle H-reflex. The mean change of passive ankle joint dorsiflexion in the combination therapy group was 11.4 (SD 4.79) degrees versus 6.1 (SD 3.09) degrees, which was significantly higher ( $\mathrm{P} 1 / 40.001)$. The mean change of plantar flexor muscle tonicity measured by the Modified Ashworth Scale in the combination therapy group was 1.6 (SD 0.5) versus 1.1 (SD 0.31) in the Bobath group ( $\mathrm{P} 1 / 40.001)$. Dorsiflexor muscle strength was also increased significantly $\left(\mathrm{P}^{1 / 40.04)}\right.$ in the combination therapy group (0.7-0.37) compared with the Bobath group (0.4-0.23). However, no significant change in the amplitude of H-reflex was found between combination therapy $(0.41-0.29)$ and Bobath (0.3-0.28) groups. This study concluded that therapy combining Bobath inhibitory technique and electrical stimulation may help to reduce spasticity effectively in stroke patients.

Ansari NN Naghdi S, ${ }^{5}$ done a clinical study to evaluate the efficacy of the Bobath approach on the excitability of the spinal alpha motor neurons in patients with post stroke spasticity. Ten subjects ranging in age from 37 through 76 years (average 60 years) with ankle plantar flexor spasticity secondary to a stroke were recruited and completed the trial. They had physiotherapy according to Bobath concept for ten treatment sessions, three days per week. Two repeated measures, one before and another after treatment were taken to quantify clinical efficacy. The effect of this type of therapy on the excitability of alpha motor neurones (aMN) was assessed by measuring the latency of the Hoffmann reflex (H-reflex) and the Hmax/Mmax ratio. The original Ashworth scale and ankle range of motion were also measured. The mean HmaxlMmax ratio on the affected side at baseline was high in the study patients. However, there were no statistically significant differences in the Hmax/Mmax ratio or in the H-reflex latency between the baseline values and those recorded after therapy intervention. Before treatment, the Hmax/Mmax ratio was significantly higher in the affected side than in the unaffected side. However, it was similar at both sides after treatment. Following treatment, the significant reduction in spasticity was clinically detected as measured with the original Ashworth scale.

Wang RY, Chen HI, Chen CY, Yang YR, ${ }^{6}$ done a randomized controlled study on Efficacy of Bobath versus orthopaedic approach on impairment and function at different motor recovery stages after stroke, to investigate the effectiveness of Bobath on stroke patients at different motor stages by comparing their treatment with orthopaedic treatment.OnTwenty-one patients with stroke with spasticity and 23 patients with stroke at relative recovery stages, found that participants with spasticity showed greater improvement in tone control (change score: $1.20+/-1.03$ versus $0.08+/-0.67, \mathrm{p}=0.006$ ), MAS (change score: $7.64+/-4.03$ versus $4.00+/-1.95, \mathrm{p}=0.011$ ), and SIS (change score: $7.30+/-6.24$ versus $1.25+/-5.33, \mathrm{p}=0.023$ ) after 20 sessions of Bobath treatment than with orthopaedic treatment. Participants with relative recovery receiving Bobath treatment showed greater improvement in MAS (change score: $6.14+/-5.55$ versus $2.77+/-9.89, \mathrm{p}=0.007$ ), BBS (change score: $19.18+/-15.94$ versus $6.85+/-5.23, \mathrm{p}=0.015$ ), and SIS scores (change score: $8.50+/-3.41$ versus $3.62+/-4.07, \mathrm{p}=0.006)$ than those with orthopaedic treatment. They concluded that Bobath or orthopaedic treatment paired with spontaneous recovery resulted in improvements in impairment and functional levels for patient with stroke. Patients benefit more from the Bobath treatment in MAS and SIS scores than from the orthopaedic treatment program regardless of their motor recovery stages. 


\section{Material and methods}

Hemiplegic's patients secondary to any type of stroke (mostly ischemic, embolic, hemorrhagic mostly MCA territory artery involment) in first stage of recovery with vital functions within normal limits aged between 40-60 years both male and female who were functionally fully dependent were divided in two groups by convenient sampling. The pre treatment functional outcome was measured by Modified Barthel Index a treatment session was given for 45 days and post treatment functional outcome was measured by Modified Barthel Index.

Group A- is a control group treated with conventional physiotherapy approach continuously for 6 days per week for the period 3 months

Group B- Experimental group Bobath approach of Neuro Rehabilitation, 6 days per week for 45days.

Before and after the treatment they were assessed using Modified Barthal Index (M.B.I.) for their functional activities.

The treatment protocols in both the groups were tailored based on the Brunstrom's stages of recovery as well as individual patient's requirement. The conventional therapy was given by the team of Neuro-Physiotherapist in the Department of Physiotherapy, Krishna Hospital karad. The Bobath Approach of stroke Rehabiliation was given solely by the researcher.

Pre-post evaluation of functional outcome was assessed blind foldedly

Due to time constrained and financial limitations only $10 \%$ of the study population were randomly selected and referred for advanced radiological investigation procedure (f-MRI/ Multiplanner scanner) for finding out the morphological changes at the site of lesion before and after the intervention.

\subsection{Outcome measures}

Modified Barthel Index measures degree of assistance required on 10 item of mobility and self care. Levels of measurement are limited to complete independence or needing assistance. It has strong interrater reliability $(0.95)$ and test re-test reliability $(0.89)$ as well as high correlations (0.74 to 0.80$)$

\subsection{Gender:}

\section{Statistical analysis}

\begin{tabular}{|l|l|l|}
\hline & Male & Female \\
\hline Group A & 15 & 15 \\
\hline Group B & 17 & 13 \\
\hline
\end{tabular}

60 subjects with stoke between age group of 38 to 72 years were taken.Out of 60 subjects, 32 included were male and 28 included were female. Group A had 15 males and 15 females and Group B had 17 males and 13 females.

\subsection{Modified Barthel Index}

4.2.1 Comparison of pre and post MBI score within groups

\begin{tabular}{|l|l|l|l|l|l|}
\hline \multirow{2}{*}{ Group } & Pre & Post & 'p' \\
\cline { 2 - 6 } & Mean & SD & Mean & SD & \\
\hline A & 5.033 & 0.88 & 14.6 & 1.83 & $<0.0001$ \\
\hline B & 5.7 & 2.17 & 14.8 & 2.14 & $<0.0001$ \\
\hline
\end{tabular}

In the present study pre-interventional mean MBI score was $5.033 \pm 0.88$ in Group A and $5.7 \pm 2.17$ in Group B, were as post-interventionally mean MBI score was $14.6 \pm 1.83$ in Group a and $14.8 \pm 2.14$ in Group B. Intra group analysis of MBI revealed statistically reduction in physical disability scores post interventionally for both the groups. This was done by using Wilcoxon matched pairs test Group A ( $<<0.0001)$, Group B $(\mathrm{p}<0.0001)$.

4.2.2 Comparison of pre-pre and post-post MBI scores in between groups.

\begin{tabular}{|l|l|l|l|l|}
\hline \multirow{2}{*}{ Group } & Pre & Post & \\
\cline { 2 - 5 } & Mean & SD & Mean & SD \\
\hline A & 5.033 & 0.88 & 14.6 & 1.83 \\
\hline B & 5.7 & 2.15 & 14.8 & 2.14 \\
\hline 'p' & 0.7538 & 0.6039 & \\
\hline
\end{tabular}

In the present study pre-interventional mean MBI score was $5.033 \pm 0.88$ in Group A and $5.7 \pm 2.15$ in Group B whereas post-interventionally mean MBI score was $14.6 \pm 1.83$ in Group A and $14.8 \pm 2.14$ in Group B respectively. Inter group analysis of MBII score was done by using Mann-Whitney test. Pre intervention analysis showed no significant difference between Group A and Group B $(p=0.7538)$. Post intervention analysis showed no significant difference between Group A and Group B ( $\mathrm{p}=0.0002)$. 


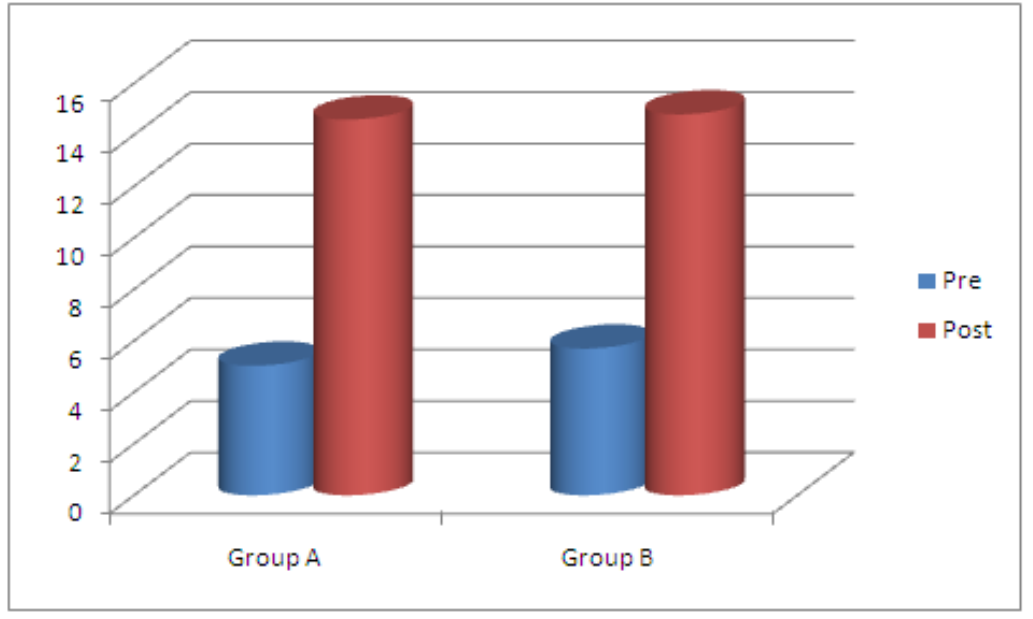

V. Discussion

This study is being conducted with the aim of finding out the effect of two different methods of physiotherapy primarily at the functional aspect (ADL) followed by structural changes at the site of lesion.

So, for all the (60) patients have shown significant improvement in their activities of daily living .A good prognosis at the affected parts of the body always due to definite positive changes at the site of lesion (basic physiocare phenomena of motor control \& motor learning of normal and affected part of the brain.)

Most of the stroke survivors in this study gained 60 to $70 \%$ of motor control and recovered all the motor skills. This supports the normal phenomena of motor control and motor learning.

To execute any kind of motor activity which are affected due to lesion at the higher centres will gain motor control of higher centres at the motor function followed by learning the skill at the various phases:

1. Cognitive phase.

2. Associative phase.

3. Autonomous phase.

Functional characteristics of affected parts of brain:

1. The affected part of brain will not be generating any kind of action potential in order to facilitate the motor recruitment of upper CNS and lower CNS.

2. The sub cortical structures will not be participating in the motor control and motor learning program of the brain.

3. The involuntary function of brain may be facilitated due to lack of initiating control of the cortex.

4. The DTR \& primitive reflex will be exaggerated.

The subjects who were treated with both the approaches either directly or indirectly influenced on functional recovery of the patients by meeting the every demand of affected part of the brain. This could be possible only when the approaches are being given at the early and tailoring the further exercises as per the need of the clients based on their clinical presentations.

\section{Conclusion}

Based on the statistical analysis and interpretations the study accepts the null hypothesis but in the group B (experimental group) the functional recovery attained by the clients were quiet earlier than Group A (control group)

\section{Limitations}

Sample size is very small for the aim and objectives of the study because of which the results found may not be generalized. The time of referal from the respective physicians were not same for both the groups always the earlier is the better.

\section{Suggestions}

Above mentioned limitations can be handled carefully by increasing the more number of homogeneous samples to generalize the result.

There could be good awareness should be created by the researcher prior to the study amongst neuro physicians for earlier referrals. 


\section{Recommendations}

All the above mentioned suggestions are recommended for future prosperous of the study.

\section{References}

[1.] Susan B O'Sullivan, Thomas J Schmitz . Physical rehabilitation. $5^{\text {th }}$ edition.

[2.] Bobath Berta. Adult hemilegia evaluation and treatment. $3^{\text {rd }}$ edition.

[3.] Bakhtiary AH et al. Does electrical stimulation reduces spasticity after stroke? Clinical Rehabilitaion. 2008 .May;22(5):418-25.U

[4.] Nazish Ansari,Soofia Naghdi. The effect of Bobath approach on the excitability of the spinal alpha motor neurons in stroke patients with muscle spasticity. Electromyography and clinical neurophysiology. January 2007.

[5.] Wang RY, Chen HI, Chen CY, Yang YR. Efficacy of Bobath versus orthopaedic approach on impairment and function at different motor recovery stages after stroke, a randomized controlled study. Clini Rehabilitation. 2005. Mar,19(2): 155-64. 